\title{
Meta
}

Journal des traducteurs

Translators' Journal

\section{Ileana CABRERA, Isabel DIÉGUEZ, Consuelo FUENZALIDA, Rosa Maria LAZO, Carolina VALDIVIESO (1987) : Factibilidad de un banco terminológico de la Pontificia Universidad católica de Chile, Santiago (Chile), PUCC, Facultad de Letras, Instituto de Letras, Departamento de Traducción, 60 p.}

\section{Jean-Claude Boulanger}

Volume 35, numéro 2, juin 1990

URI : https://id.erudit.org/iderudit/002119ar

DOI : https://doi.org/10.7202/002119ar

Aller au sommaire du numéro

Éditeur(s)

Les Presses de l'Université de Montréal

ISSN

0026-0452 (imprimé)

1492-1421 (numérique)

Découvrir la revue

Citer ce compte rendu

Boulanger, J.-C. (1990). Compte rendu de [Ileana CABRERA, Isabel DIÉGUEZ, Consuelo FUENZALIDA, Rosa Maria LAZO, Carolina VALDIVIESO (1987) : Factibilidad de un banco terminológico de la Pontificia Universidad católica de Chile, Santiago (Chile), PUCC, Facultad de Letras, Instituto de Letras,

Departamento de Traducción, 60 p.] Meta, 35(2), 432-434.

https://doi.org/10.7202/002119ar d'utilisation que vous pouvez consulter en ligne. 
Ileana CABRERA, Isabel DIÉGUEZ, Consuelo FUENZALIDA, Rosa Maria LAZO, Carolina VALDIVIESO (1987): Factibilidad de un banco terminológico de la Pontificia Universidad católica de Chile, Santiago (Chile), PUCC, Facultad de Letras, Instituto de Letras, Departamento de Traducción, 60 p.

Dans ce petit ouvrage, qui est ici l'objet d'un compte rendu descriptif et non pas critique, les auteures présentent l'étude de faisabilité d'une banque de terminologie au Chili. Quatre parties structurent le rapport: 1. Présentation du problème; 2. Antécédents théo-. riques; 3 . Modèle d'analyse et méthodologie; 4 . Présentation des résultats. Je passe successivement chacune en revue et $\mathrm{j}$ 'en extrais les éléments essentiels.

En avant-propos, les responsables de la recherche rappellent fort justement que la terminologie est une discipline qui doit absolument être intégrée au cursus universitaire de tout programme de traduction qui se veut à l'avant-garde de cette profession. Dans la même foulée, elles constatent que la production terminologique croissante crée le besoin d'un vaste réservoir de données comme peut l'être une banque de terminologie. On peut ainsi y consigner la masse de termes de plus en plus nombreux auxquels les traducteurs et les terminologues doivent recourir dans leur travail professionnel. Afin de satisfaire l'appétit des professionnels de la langue, ces banques doivent constamment être enrichies et mises à jour. Devant les nombreuses interrogations que soulève la mise en place d'une banque de terminologie et devant les dépenses qu'elle entraîne, il est souhaitable pour les Chiliens de suivre une démarche étapiste. Le cheminement retenu propose d'abord une étude des besoins terminologiques, puis l'entreprise de faisabilité elle-même, enfin la pla-. nification de l'implantation de la banque de la manière la plus rationnelle et la plus économique possible.

Les responsables du projet ont également mis de l'avant l'importance de comprendre la documentation scientifique et technique rédigée en langue étrangère, particulièrement en anglais, et que les étudiants et les professeurs doivent consulter tout au long de leur formation et de leurs recherches.

La première partie du rapport explique le phénomène d'accroissement des termes qui augmentent plus rapidement que les mots de la langue générale. On y traite des liens 
entre la néologie et les technologies de pointe, du savoir-faire en matière de création de termes, du grand nombre de publications scientifiques qui naît chaque année, de la circulation de l'information en plusieurs langues. Les objectifs du projet sont ensuite détaillés: il s'agit d'abord de diagnostiquer les besoins terminologiques des étudiants, des enseignants et des professionnels de la traduction, puis de voir si une banque de terminologie est vraiment le remède le plus efficace pour faire face à la musique.

Dans le chapitre sur les antécédents théoriques, les auteures procèdent à un rapide tour d'horizon bibliographique des ouvrages portant sur la terminologie et la lexicographie, sur les banques de terminologie et sur les LSP en général. Elles présentent les conceptions de la terminologie en Autriche, en Russie, en Tchécoslovaquie et au Canada. C'est sur l'école canado-québécoise que s'appuient les chercheuses pour définir quatre concepts fondamentaux: «terminologie», «terme», «banque de terminologie», «besoins terminologiques».

Le chapitre 3 présente le profil de la recherche qui est axée sur la description des attentes des étudiants et des professeurs en matière de terminologie multilingue, tant pour l'apprentissage, pour les premiers, que pour la recherche et l'enseignement, pour les seconds. Trois centres d'intérêt retiennent l'attention: 1. La nécessité pour tous de lire la documentation rédigée en langue étrangère. 2. L'évaluation des difficultés terminologiques dans les textes techniques et scientifiques étrangers. 3. La création de dictionnaires terminologiques et l'évaluation de leur utilisation. La méthodologie explique tout le processus de l'enquête sur le campus universitaire: choix des répondants, secteurs d'intervention, etc. De nombreux tableaux statistiques éclairent les explications des auteures. Les questionnaires sont reproduits en annexe.

Les résultats de la recherche sont précisés au chapitre 4. Retenons de l'ensemble que la majorité des étudiants trouve qu'il est difficile de lire des textes en langues étrangères parce qu'ils ne peuvent avoir accès aux définitions des termes dans leur champ de spécialité. Un certain nombre d'entre eux souhaitent qu'il y ait une augmentation des cours de langue étrangère. Sauf dans le secteur de la traduction, les étudiants et les professeurs ignorent souvent qu'il existe des dictionnaires terminologiques dans leur domaine de compétence.

Une conclusion en sept points résume bien le contenu du rapport:

1. Les titres en langue étrangère représentent $44 \%$ des ouvrages contenus dans les bibliographies des cours.

2. Parmi ces titres, l'anglais domine largement les autres langues.

3. En raison de leur niveau de compétence assez bas, les étudiants lisent peu en langue étrangère.

4. Tous sont d'accord pour reconnaître l'existence de difficultés terminologiques dans les textes. Les traducteurs sont mieux préparés à résoudre ces difficultés.

5. Sauf en traduction, les enseignants proposent de traduire les textes les plus importants pour leurs cours. Quant aux étudiants, ils croient pouvoir résoudre le problème en augmentant les cours de langue spécialisée. Les groupes de traducteurs souhaitent la mise sur pied de banques de terminologie.

6. Sauf en traduction, on ignore l'existence de dictionnaires terminologiques. Lorsqu'on connaitt leur existence, on semble ne pas savoir comment s'en servir.

7. L'élaboration de dictionnaires traditionnels est privilégiée par les gens qui ne sont pas en traduction (étudiants : 68,6\%, professeurs : $63,4 \%$ ). Les langagiers préfèrent le dictionnaire automatisé $(67,5 \%$ des répondants).

Étant donné les opinions très divergentes des personnes questionnées, les auteures proposent que l'on crée une banque de terminologie réservée au secteur de la traduction dans un premier temps. Par la suite, on pourra étendre la prestation des services à 
l'ensemble de la communauté universitaire. À long terme, une banque de terminologie ouvrirait la voie à la création d'un centre de terminologie à la PUCC, centre qui serait d'une grande utilité pour l'ensemble du pays. 